\title{
A Parallel Prefiltering Approach for the Identification of a Biased Sinusoidal Signal: Theory and Experiments
}

\author{
Boli Chen ${ }^{1}$, Gilberto Pin ${ }^{2}$, Wai M. Ng ${ }^{3}$, S. Y. Ron Hui ${ }^{1,3}$, and Thomas Parisini ${ }^{1,4 *}$ \\ ${ }^{1}$ Dept. of Electrical and Electronic Engineering, Imperial College London, UK \\ ${ }^{2}$ Electrolux Professional S.p.A., Pordenone, Italy \\ ${ }^{3}$ Dept. of Electrical and Electronic Engineering, The University of Hong Kong, HK \\ ${ }^{4}$ Dept. of Engineering and Architecture, University of Trieste, Italy
}

\begin{abstract}
SUMMARY
The problem of estimating the amplitude, frequency and phase of an unknown sinusoidal signal from a noisy biased measurement is addressed in this paper by a family of parallel pre-filtering schemes. The proposed methodology consists in using a pair of linear filters of specified order to generate a suitable number of auxiliary signals that are used to estimate - in an adaptive way - the frequency, the amplitude and the phase of the sinusoid. Increasing the order the pre-filters improves the noise immunity of the estimator, at the cost of an increase of the computational complexity. Among the whole family of estimators realizable by varying the order of the filters, the simple parallel pre-filters of order $2+2$ and $3+3$ are discussed in detail, being the most attractive from the implementability point of view. The behavior of the two algorithms with respect to bounded external disturbances is characterized by Input-to-State Stability arguments. Finally, the effectiveness of the proposed technique is shown both by comparative numerical simulations and by a real experiment addressing the estimation of the frequency of the electrical mains from a noisy voltage measurement. Copyright (c) 2015 John Wiley \& Sons, Ltd.
\end{abstract}

Received ...

\section{INTRODUCTION}

Methodologies for the on-line identification of a sinusoidal signal from uncertain measurements are widely employed in many engineering applications such as active noise cancellation, vibrations monitoring in mechanical system and periodic disturbance rejection (see $[1,2,3,4,5]$ and the references cited therein), to mention a few. A variety of techniques has been presented in the literature for estimating the unknown sinusoids in terms of estimating the amplitude, the frequency and the phase (AFP), such as phase-lock-loop (PLL), adaptive notch filter (ANF) and state variable filtering (see, for instance $[6,7,8,9,10]$ ).

Recently significant research efforts have been devoted to the AFP estimation in presence of an unknown constant bias, which occurs in several practical applications, such as the offsets in physical transducers and A/D converters. In [11] and [12], the conventional PLL and ANF techniques are augmented by an additional integrator loop in charge of estimating the bias term. The Orthogonal Signal Generator-Second Order Generalized Integrator (OSG-SOGI) architecture represents an alternative solution to cope with a biased sinusoidal signal (see [13]). Moreover, in [14], a bank of adapted frequency-locked-loops (AFPLL) based on the third order generalized integrator OSG (OSG-TOGI) (see [15]) are exploited to address the multi-sinusoidal estimation problem. However, the available stability results obtained by averaging theory only ensure local convergence of the

*Correspondence to: Thomas Parisini. Email address: (t.parisini@gmail.com)

Copyright (c) 2015 John Wiley \& Sons, Ltd.

Prepared using acsauth.cls [Version: 2010/03/27 v2.00] 
OSG-TOGI estimator. The same authors introduced in [16] a simple recursive frequency estimator based on the modulating functions, that may easily annihilate the bias in the measurement.

In [17] an adaptive fourth-order estimator is proposed with aim of improving the robustness of the original method presented by the same authors in [18]. By adopting a gradually decreasing adaptive gains, the effect of high-frequency noise is attenuated, thus leading to accurate estimates in steady state.

Techniques based on adaptive observers have also been proposed in the recent literature (see [19, 20, 21, 22, 23, 24]). In particular, [24] deals with a "dual-mode" estimation scheme, incorporating a switching algorithm (depending on the real-time excitation level) into an adaptive observer-based sinusoidal estimator. In the spirit of the preliminary results presented in [23], the robustness in presence of structured and unstructured uncertainties are addressed by Input-to-state stability (ISS) arguments. A notable feature of adaptive observer schemes is the possibility of carrying out multi-sinusoidal estimation by expanding the dynamic model with a suitable system transformation (see, for example, [19, 21, 25, 26]).

In the papers [27, 28, 23], a set of cascaded first-order low-pass (LP) filters, called "pre-filter" is exploited with the aim of both cancelling the effect of structured "time-polynomial" perturbations (such as bias and linear drift) and obtaining auxiliary signals that can be exploited to estimate the frequency and the amplitude of the sinusoid. These auxiliary signals can be used either directly $[27,28]$ or indirectly [23] to estimate the unknown frequency and the amplitude of the measured noisy sinusoid with high noise immunity.

Nevertheless, while in $[23,27,28]$ the pre-filter was conceived as a cascade of first-order LP filters, in the present paper we propose to use a parallel pre-filtering scheme, where two LP filters with different poles are used to generate auxiliary signals from which the needed information is extracted. This enhanced structure allows to simplify the adaptation law with respect to $[28,23]$, while maintaining robustness with respect to bounded measurement perturbations. Compared with the well-behaved algorithms proposed in [17, 18, 29] - that are based on a second-order prefilter - we will show by numerical simulations that the use two parallel pre-filters may lead to improved steady state and transient performances. Moreover, the use of two pre-filters allows the squared-frequency parameter to be adapted directly, without resorting to intermediate variables as in $[17,18,29]$, thus simplifying the structure of the estimator. As in the case of the seminal papers $[17,18]$, we will characterize the behaviour of the estimator in presence of a bounded additive measurement disturbance. While in [22,29] a leakage correction term is added to the adaptation law to prevent the parameter drift in case of external perturbation, we will prove that the proposed method is inherently ISS with respect to the exogenous disturbance. The ISS analysis will also make it possible to obtain useful tuning guidelines, since the dissipation rate and the ISS-asymptotic-gain are both expressed in terms of the estimator's parameters. Finally, the effectiveness of proposed method is shown by several comparative simulations, as well as by experimental results with a dSpace system on a typical micro-grid voltage signal and compared with two recent AFP approaches presented in [11] and [13], respectively.

\section{THE PARALLEL PRE-FILTERING SCHEME}

Consider an unknown sinusoidal signal corrupted by a norm-bounded time-varying disturbance $d(t):|d(t)|<\bar{d}$, with $\bar{d} \geq 0$ a worst-case bound known conservatively:

$$
y(t)=v(t)+d(t)
$$

in which

$$
v(t)=A_{0}+A \sin (\vartheta(t)), \quad \dot{\vartheta}(t)=\omega^{*}, \vartheta(0)=\vartheta_{0}
$$

where $A_{0}$ represents a constant offset (bias) term.

To estimate the frequency, the phase and the amplitude of the original sinusoid, the noisy measurement $y(t)$ is filtered by a pair of low-pass linear filters, indexed by $k \in\{1,2\}$. Let us denote by $\hat{\mathbf{x}}_{k}(t)=\left[\hat{x}_{k, 1}(t) \cdots \hat{x}_{k, n}(t)\right]^{\top} \in \mathbb{R}^{n}, k \in\{1,2\}$ the state vector of a the $k$-th filter, driven by $y(t)$ 
and evolving from arbitrary initial conditions:

$$
\left\{\begin{array}{l}
\dot{\hat{\mathbf{x}}}_{k}(t)=\mathbf{F}_{k} \hat{\mathbf{x}}_{k}(t)+\mathbf{g}_{k} y(t), \quad \forall k=1,2, \quad t \in \mathbb{R}_{\geq 0} \\
\hat{\mathbf{x}}_{k}(0)=\hat{\mathbf{x}}_{k_{0}}
\end{array}\right.
$$

where $\mathbf{F}_{k} \in \mathbb{R}^{n \times n}, \forall k \in\{1,2\}$ is given by

$$
\mathbf{F}_{k}=\left[\begin{array}{ccccc}
-\lambda_{k} & 0 & \cdots & \cdots & 0 \\
& & & & \\
\beta_{k} \lambda_{k} & -\lambda_{k} & \ddots & \ddots & \vdots \\
0 & \ddots & \ddots & \ddots & \vdots \\
\vdots & \ddots & \ddots & \ddots & 0 \\
0 & \cdots & 0 & \beta_{k} \lambda_{k} & -\lambda_{k}
\end{array}\right],
$$

and

$$
\mathbf{g}_{k}=\left[\begin{array}{llll}
\beta_{k} \lambda_{k} & 0 & \cdots & 0
\end{array}\right]^{\top}, \forall k \in\{1,2\} .
$$

The parameters $\lambda_{1}, \lambda_{2} \in \mathbb{R}_{>0}$, with $\lambda_{1} \neq \lambda_{2}$ represent the poles of low-pass filters, while $\beta_{1}, \beta_{2} \in$ $(0,1]$ act as damping gains. For the sake of further analysis, let us introduce the united state vector $\hat{\mathbf{x}}(t)=\left[\hat{\mathbf{x}}_{1}(t) \hat{\mathbf{x}}_{2}(t)\right]^{\top}$ which is governed by the following differential equation in virtue of (3):

$$
\dot{\hat{\mathbf{x}}}(t)=\mathbf{A}_{\lambda, \beta} \hat{\mathbf{x}}(t)+\mathbf{b}_{\lambda, \beta} y(t), \quad t \in \mathbb{R}_{\geq 0}
$$

with $\hat{\mathbf{x}}(0)=\left[\hat{\mathbf{x}}_{1_{0}}^{\top}, \hat{\mathbf{x}}_{2_{0}}^{\top}\right]^{\top}$ and

$$
\mathbf{A}_{\lambda, \beta}=\left[\begin{array}{cc}
\mathbf{F}_{1} & 0 \\
0 & \mathbf{F}_{2}
\end{array}\right], \quad \mathbf{b}_{\lambda, \beta}=\left[\begin{array}{l}
\mathbf{g}_{1} \\
\mathbf{g}_{2}
\end{array}\right],
$$

Now, in order to describe how the state vectors of the filters can be used to obtain an estimate of the frequency of the biased sinusoid $v(t)$ (see (2)), we will characterize the asymptotic behaviour of the filters' states, showing that in nominal condition they tend to a stationary sinusoidal regime.. To this end, we will denote by $\mathbf{x}_{k}(t), k \in\{1,2\}$ two "virtual" state vectors (i.e., not implemented in practice but instrumental to carry out the stability analysis), obeying the dynamics (3) driven by the disturbance-free signal $v(t)$ (not measurable) in place of $y(t)$. Moreover, let $\mathbf{x}(t)=\left[\mathbf{x}_{1}(t) \mathbf{x}_{2}(t)\right]^{\top}$ be the combined virtual state vector, that is the noise-free counterpart of the vector $\hat{\mathbf{x}}(t)$ defined above. In view of the realization of the two filters, it holds that

$$
\mathbf{c}^{\top} \mathbf{F}_{k}^{q} \mathbf{g}_{k}=0, \forall q \in\{0, \ldots, n-2\},
$$

where $\mathbf{c}=\left[\begin{array}{llll}0 & \cdots & 0 & 1\end{array}\right]^{\top}$. In view of (5), the derivatives of the $n$-th state variables $x_{1, n}(t)$ and $x_{2, n}(t)$ of the two filters can be expressed as:

$$
\begin{aligned}
& \frac{d^{q}}{d t^{q}} x_{k, n}(t)=\mathbf{c}^{\top} \mathbf{F}_{k}^{q} \mathbf{x}_{k}(t), q \in\{1, \ldots, n-1\}, \\
& \frac{d^{n}}{d t^{n}} x_{k, n}(t)=\mathbf{c}^{\top} \mathbf{F}_{k}^{n-1}\left(\mathbf{F}_{k} \mathbf{x}_{k}(t)+\mathbf{g}_{k} v(t)\right) .
\end{aligned}
$$

Denoting by $G_{k, n}(s), k \in\{1,2\}$ the transfer functions of the filters in the Laplace domain from the input signal to the $n$-th state variables: $\mathcal{L}\left[x_{k, n}\right](s)=G_{k, n}(s) \mathcal{L}[v](s), k \in\{1,2\}$, we have that

$$
G_{k, n}(s)=\frac{\lambda_{k}^{n} \beta_{k}^{n}}{\left(s+\lambda_{k}\right)^{n}} .
$$

Neglecting the initial conditions of the internal filter's states, the Laplace transform of the $n$-th state variables can be expressed as:

$$
\mathcal{L}\left[x_{k, n}\right](s)=G_{k, n}(s) A \frac{s \sin \left(\vartheta_{0}\right)+\omega^{*} \cos \left(\vartheta_{0}\right)}{s^{2}+\omega^{* 2}}+G_{k, n}(s) \frac{A_{0}}{s} .
$$


and then the transform of the $q$-th derivative of $x_{k, n}$ is

$$
\mathcal{L}\left[\frac{d^{q} x_{k, n}}{d t^{q}}\right](s)=G_{k, n}(s) A \frac{s \sin \left(\vartheta_{0}\right)+\omega^{*} \cos \left(\vartheta_{0}\right)}{s^{2}+\omega^{* 2}} s^{q}+G_{k, n}(s) A_{0} s^{q-1} .
$$

Thus, it is readily seen that in the time-domain, the derivatives $\frac{d^{q}}{d t^{q}} x_{k, n}(t), 1 \leq q \leq n$ own the asymptotic sinusoidal steady-state behavior as $t \rightarrow \infty$ :

$$
\frac{d^{q}}{d t^{q}} x_{k, n}(t) \approx A_{k, q} \sin \vartheta_{k, q}(t), \forall t>>0 k \in\{1,2\}
$$

where

$$
\begin{aligned}
& A_{k, q}=A \omega^{* q}\left|G_{k, n}\left(j \omega^{*}\right)\right|, \\
& \vartheta_{k, q}(t)=\vartheta(t)+\angle G_{k, n}\left(j \omega^{*}\right)+\frac{\pi}{2} q .
\end{aligned}
$$

Note that the derivative signals (7) can be directly computed from the filters' states by (6) and can be used as auxiliary signals to retrieve the frequency, the amplitude and the phase of the input sinusoid.

In the next Section, we will first characterize the stability properties of a generalized parallel-prefiltering based estimation scheme, where each filter has order equal to $n$; the order can be reduced to a $3+3$ configuration characterized by the same unified convergence analysis. Then, in Sec. 4 , we will further reduce the order yielding to a simplified $2+2$ scheme of which the stability analysis has to be carried out in a different way.

\section{METHOD 1: ORDER $n+n$ PRE-FILTERING-BASED FREQUENCY ESTIMATOR}

\subsection{Underlying idea}

The order $n$ of the pre-filters is fixed by the designer depending on the performance requested to the estimator. Indeed, we will show that, at the cost of increasing of the order of the pre-filter, the algorithm can be modified to get an enhanced noise immunity.

Without loss of generality, let us start from considering a pre-filtering stage having two prefilters each of order $n$, where $n \geq 3$. In correspondence to the available auxiliary vectors $\hat{\mathbf{z}}_{1}^{\prime}(t)=$ $\left[\frac{d^{n-2}}{d t^{n-2}} \hat{x}_{1, n}(t) \frac{d^{n-2}}{d t^{n-2}} \hat{x}_{2, n}(t)\right]^{\top}$ and $\hat{\mathbf{z}}_{2}^{\prime}(t)=\left[\frac{d^{n}}{d t^{n}} \hat{x}_{1, n}(t) \frac{d^{n}}{d t^{n}} \hat{x}_{2, n}(t)\right]^{\top}$, formed by the derivatives of the internal pre-filter's states, we introduce the counterparts in the noise-free condition: $\mathbf{z}_{1}^{\prime}(t)=$ $\left[\frac{d^{n-2}}{d t^{n-2}} x_{1, n}(t) \frac{d^{n-2}}{d t^{n-2}} x_{2, n}(t)\right]^{\top}$ and $\mathbf{z}_{2}^{\prime}(t)=\left[\frac{d^{n}}{d t^{n}} x_{1, n}(t) \frac{d^{n}}{d t^{n}} x_{2, n}(t)\right]^{\top}$, which own the sinusoidal equilibrium $\overline{\mathbf{z}}_{1}^{\prime}(t), \overline{\mathbf{z}}_{2}^{\prime}(t)$. For simplicity, let us create the combined vectors $\hat{\mathbf{z}}^{\prime}(t)=\left[\hat{\mathbf{z}}_{1}^{\prime}(t) \hat{\mathbf{z}}_{2}^{\prime}(t)\right]^{\top}$, $\overline{\mathbf{z}}^{\prime}(t)=\left[\overline{\mathbf{z}}_{1}^{\prime}(t) \overline{\mathbf{z}}_{2}^{\prime}(t)\right]^{\top}$, where $\tilde{\mathbf{z}}_{k}^{\prime}(t)=\hat{\mathbf{z}}_{k}^{\prime}(t)-\overline{\mathbf{z}}_{k}^{\prime}(t), \forall k \in\{1,2\}$ represents the error vector in presence of $d(t)$. The vector of auxiliary signals $\hat{\mathbf{z}}^{\prime}(t)$ can be expressed directly in terms of the available input $y(t)$ and the $n$-th order filter's states, $\hat{\mathbf{x}}_{1}(t)$ and $\hat{\mathbf{x}}_{2}(t)$ :

$$
\hat{\mathbf{z}}^{\prime}(t)=\Lambda_{n}\left[\begin{array}{lll}
y(t) & \hat{\mathbf{x}}_{1}(t)^{\top} & \hat{\mathbf{x}}_{2}(t)^{\top}
\end{array}\right]^{\top},
$$

with

$$
\Lambda_{n}=\left[\begin{array}{ccc}
0 & \mathbf{c}^{\top} \mathbf{F}_{1}^{n-2} & 0 \\
0 & 0 & \mathbf{c}^{\top} \mathbf{F}_{2}^{n-2} \\
\mathbf{c}^{\top} \mathbf{F}_{1}^{n-1} \mathbf{g}_{1} & \mathbf{c}^{\top} \mathbf{F}_{1}^{n} & 0 \\
\mathbf{c}^{\top} \mathbf{F}_{2}^{n-1} \mathbf{g}_{2} & 0 & \mathbf{c}^{\top} \mathbf{F}_{2}^{n}
\end{array}\right]
$$

Moreover, the stationary sinusoids $\overline{\mathbf{z}}_{1}^{\prime}(t), \overline{\mathbf{z}}_{2}^{\prime}(t)$ defined in (24) satisfy the equality

$$
\Omega^{*} \overline{\mathbf{z}}_{1}^{\prime}(t)+\overline{\mathbf{z}}_{2}^{\prime}(t)=0 .
$$

We propose to obtain the unknown frequency of the measured signal by enforcing the above constraint on the estimated one. To this end, the following gradient-based adaptation law represents 
the core of the methodology, which exploits the available pre-filtered signals $\hat{\mathbf{z}}_{1}^{\prime}(t), \hat{\mathbf{z}}_{2}^{\prime}(t)$ in place of the unavailable pure sinusoids $\overline{\mathbf{z}}_{1}^{\prime}(t)$ and $\overline{\mathbf{z}}_{2}^{\prime}(t)$ :

$$
\dot{\hat{\Omega}}(t)=-\mu_{2} \hat{\mathbf{z}}_{1}^{\prime}(t)^{\top}\left(\hat{\Omega}(t) \hat{\mathbf{z}}_{1}^{\prime}(t)+\hat{\mathbf{z}}_{2}^{\prime}(t)\right) .
$$

Besides the frequency-adaptation mechanism just described, the pre-filter-based AFP scheme also involves an amplitude and phase estimation method that will be described later on in Section 5.

\subsection{Stability Analysis of the Frequency Estimator}

In order to characterize the stability property of the frequency estimation system (9) and (10), let us first address the stability of the pre-filter dynamics. Observe that there exists an (unknown) initial filter's state $\mathbf{x}(0)=\overline{\mathbf{x}}_{0}$ giving rise to a "virtual" pre-filter state trajectory $\overline{\mathbf{x}}(t)$ whose projection on the subspace containing $\mathbf{z}(t)$ matches the stationary sinusoidal behavior since the beginning, when driven by the unperturbed sinusoid $v(t)$, that is:

$$
\overline{\mathbf{x}}(t): \boldsymbol{\Lambda}_{n}\left[v(t), \overline{\mathbf{x}}(t)^{\top}\right]^{\top}=\overline{\mathbf{z}}^{\prime}(t) \triangleq\left[\begin{array}{c}
\overline{\mathbf{z}}_{1}^{\prime}(t) \\
\overline{\mathbf{z}}_{2}^{\prime}(t)
\end{array}\right], \forall t \in \mathbb{R}_{\geq 0}
$$

The dynamics of the error vector $\tilde{\mathbf{x}}(t)=\hat{\mathbf{x}}(t)-\overline{\mathbf{x}}(t)$ are governed by

$$
\left\{\begin{array}{l}
\dot{\tilde{\mathbf{x}}}(t)=\mathbf{A}_{\lambda, \beta} \tilde{\mathbf{x}}(t)+\mathbf{b}_{\lambda, \beta} d(t), \quad t \in \mathbb{R}_{\geq 0} \\
\tilde{\mathbf{x}}(0)=\hat{\mathbf{x}}_{0}-\overline{\mathbf{x}}_{0} .
\end{array}\right.
$$

Since $\mathbf{A}_{\lambda, \beta}$ is Hurwitz, it is easy to prove that the error dynamics ISS with respect to $\bar{d}$. Assume the corresponding ISS asymptotic gain is $\gamma_{x}^{\prime}(s)$, hence, for any arbitrary $\nu^{\prime} \in \mathbb{R}_{>0}$ and for any finitenorm initial error $\tilde{\mathbf{x}}_{0}$, the error vector $\tilde{\mathbf{x}}(t)$ will enter in a closed ball of radius $\gamma_{x}^{\prime}(\bar{d})+\nu^{\prime}$ in finite time.

The ISS of $\tilde{\mathbf{x}}(t)$ implies that $\tilde{\mathbf{z}}^{\prime}(t)$ is ISS and that the error trajectory will enter in finite-time in a closed ball of radius $\gamma_{z}^{\prime}(\bar{d})+\delta^{\prime}$ centered at the origin, where

$$
\gamma_{z}^{\prime}(s)=\bar{\lambda}_{n}\left(\gamma_{x}^{\prime}(s)+s\right), \forall s \in \mathbb{R}_{\geq 0}, \delta^{\prime}=\bar{\lambda}_{n} v^{\prime}
$$

with $\bar{\lambda}_{n}=\left\|\Lambda_{n}\right\|$.

Defining the frequency estimation error $\tilde{\Omega}(t) \triangleq \hat{\Omega}(t)-\Omega^{*}$, and applying the identity $\overline{\mathbf{z}}_{2}^{\prime}(t)=$ $-\Omega^{*} \overline{\mathbf{z}}_{1}^{\prime}(t)$, the dynamics of the frequency estimation error $\tilde{\Omega}(t)$ evolve according to

$$
\begin{aligned}
\dot{\tilde{\Omega}}(t) & =-\mu_{2}\left(\overline{\mathbf{z}}_{1}^{\prime}(t)+\tilde{\mathbf{z}}_{1}^{\prime}(t)\right)^{\top}\left(\hat{\Omega}(t)\left(\overline{\mathbf{z}}_{1}^{\prime}(t)+\tilde{\mathbf{z}}_{1}^{\prime}(t)\right)-\Omega^{*} \overline{\mathbf{z}}_{1}^{\prime}(t)+\tilde{\mathbf{z}}_{2}^{\prime}(t)\right) \\
& =-\mu_{2} \tilde{\Omega} \overline{\mathbf{z}}_{1}^{\prime}(t)^{\top} \overline{\mathbf{z}}_{1}^{\prime}(t)+\mu_{2} \tilde{\Omega} f_{1}\left(t, \tilde{\mathbf{z}}^{\prime}\right)+\mu_{2} f_{2}\left(t, \tilde{\mathbf{z}}^{\prime}\right)
\end{aligned}
$$

where

$$
\begin{aligned}
& f_{1}^{\prime}\left(t, \tilde{\mathbf{z}}^{\prime}\right)=-2 \overline{\mathbf{z}}_{1}^{\prime}(t)^{\top} \tilde{\mathbf{z}}_{1}^{\prime}(t)-\tilde{\mathbf{z}}_{1}^{\prime}(t)^{\top} \tilde{\mathbf{z}}_{1}^{\prime}(t) \\
& f_{2}^{\prime}\left(t, \tilde{\mathbf{z}}^{\prime}\right)=\Omega^{*}\left(f_{1}\left(t, \tilde{\mathbf{z}}^{\prime}\right)+\tilde{\mathbf{z}}_{1}^{\prime}(t)^{\top} \overline{\mathbf{z}}_{1}^{\prime}(t)\right)-\hat{\mathbf{z}}_{1}^{\prime}(t)^{\top} \tilde{\mathbf{z}}_{2}^{\prime}(t) .
\end{aligned}
$$

Note that the functions $f_{1}^{\prime}(t, \tilde{\mathbf{z}})$ and $f_{2}^{\prime}(t, \tilde{\mathbf{z}})$ verify $f_{1}^{\prime}(t, 0)=0, f_{2}^{\prime}(t, 0)=0$ for all $t \in \mathbb{R}_{\geq 0}$. Moreover, owing to the boundedness of $\mathbf{z}(t)$, there exist two $\mathcal{K}_{\infty}$-functions $\sigma_{1}^{\prime}(\cdot)$ and $\sigma_{2}^{\prime}(\cdot)$ such that

$$
\left|f_{1}^{\prime}\left(t, \tilde{\mathbf{z}}^{\prime}\right)\right| \leq \sigma_{1}^{\prime}\left(\left|\tilde{\mathbf{z}}^{\prime}\right|\right), \quad\left|f_{2}^{\prime}\left(t, \tilde{\mathbf{z}}^{\prime}\right)\right| \leq \sigma_{2}^{\prime}\left(\left|\tilde{\mathbf{z}}^{\prime}\right|\right) .
$$

Then, a further bound of $\dot{\tilde{\Omega}}(t)$ is achieved:

$$
\dot{\tilde{\Omega}}(t) \leq-\mu_{2}\left(\overline{\mathbf{z}}_{1}^{\prime}(t)^{\top} \overline{\mathbf{z}}_{1}^{\prime}(t)-\sigma_{1}^{\prime}\left(\left|\tilde{\mathbf{z}}^{\prime}\right|\right)\right) \tilde{\Omega}(t)+\mu_{2} \sigma_{2}^{\prime}\left(\left|\tilde{\mathbf{z}}^{\prime}\right|\right)
$$

The following assumption is needed to prove the convergence of the estimation error. 


\section{Assumption 1}

The signal $\overline{\mathbf{z}}_{1}^{\prime}(t)$ is persistently exciting in the sense that there exist a constant $\varepsilon>0$ such that

$$
\overline{\mathbf{z}}_{1}^{\prime}(t)^{\top} \overline{\mathbf{z}}_{1}^{\prime}(t) \geq \varepsilon, \forall t>0
$$

In the following derivations we show that Assumption 1 is verified under a suitable choice of $\lambda_{1}$ and $\lambda_{2}$ in the nominal (noise-free) condition. Let us first introduce the operator $G_{k, n}(s)=$ $\frac{\lambda_{k}^{n} \beta_{k}^{n}}{\left(s^{+} \lambda_{k}\right)^{n}}, \forall k \in\{1,2\}$, and then expand $\overline{\mathbf{z}}_{1}^{\prime}(t)^{\top} \overline{\mathbf{z}}_{1}^{\prime}(t)$ by

$$
\begin{aligned}
\overline{\mathbf{z}}_{1}^{\prime}(t)^{\top} \overline{\mathbf{z}}_{1}^{\prime}(t) & =\left[\frac{d^{n-2} \bar{x}_{1, n}}{d t^{n-2}}(t)\right]^{2}+\left[\frac{d^{n-2} \bar{x}_{2, n}}{d t^{n-2}}(t)\right]^{2} \\
& =\sum_{k=1}^{2} A_{k, n-2} \sin ^{2}\left(\vartheta(t)+\phi_{k}+\frac{\pi}{2}(n-2)\right)
\end{aligned}
$$

in which $A_{k, n-2}=A \omega^{* n-2}\left|G_{k, n}\left(j \omega^{*}\right)\right|, \phi_{k}$ represents the phase of $G_{k, n}\left(j \omega^{*}\right)$. After some algebra, we get

$$
\phi_{1}=n \arctan \left(-\frac{\omega^{*}}{\lambda_{1}}\right), \quad \phi_{2}=n \arctan \left(-\frac{\omega^{*}}{\lambda_{2}}\right) .
$$

Thanks to the fact that $\left|\phi_{1}-\phi_{2}\right|<\frac{n \pi}{2}, \forall \omega^{*}>0, \overline{\mathbf{z}}_{1}^{\prime}(t)^{\top} \overline{\mathbf{z}}_{1}^{\prime}(t)$ is strictly positive for $t>0$ if and only if $\left|\phi_{1}-\phi_{2}\right| \neq k \pi, k \in[0,1,2, \ldots .\lfloor n / 2\rfloor), \forall \omega^{*} \in \mathbb{R}_{>0}$ with $\lfloor\cdot\rfloor$ the floor operator. It is readily seen that when $\lambda_{1}$ and $\lambda_{2}$ are distinct, i.e. $\phi_{1} \neq \phi_{2}, \forall \omega^{*}>0$. In the following, we will show that by choosing the pre-filters parameters such that

$$
\lambda_{1}^{2}+\lambda_{2}^{2}-\left(2+4 \alpha^{2}\right) \lambda_{1} \lambda_{2}<0
$$

in which $\alpha=\tan (\pi / n)$, then the condition $\left|\phi_{1}-\phi_{2}\right| \neq k \pi, k \in[1,2, \ldots\lfloor n / 2\rfloor), \forall \omega^{*}>0$ is satisfied as well. Let us make the hypothesis that there exists $\omega^{*} \in \mathbb{R}_{>0}$, such that $\left|\phi_{1}-\phi_{2}\right|=k \pi$. Such hypothesis holds if and only if

$$
\arctan \left(\frac{\omega^{*}}{\lambda_{1}}\right)-\arctan \left(\frac{\omega^{*}}{\lambda_{2}}\right)= \pm \frac{k \pi}{n} .
$$

Taking $\tan (\cdot)$ on both hand side of the above equation, and owing to the property of trigonometric functions, we arrive at

$$
\left(\frac{\omega^{*}}{\lambda_{1}}-\frac{\omega^{*}}{\lambda_{2}}\right) /\left(1+\frac{\omega^{* 2}}{\lambda_{1} \lambda_{2}}\right)= \pm \tan (k \pi / n)
$$

which can be rearranged to

$$
\tan (k \pi / n) \omega^{* 2} \pm\left(\lambda_{1}-\lambda_{2}\right) \omega^{*}+\tan (k \pi / n) \lambda_{1} \lambda_{2}=0 .
$$

Note that equation (17) does not admit any real root in the variable $\omega^{*}$ as long as $\lambda_{1}^{2}+\lambda_{2}^{2}-(2+$ $\left.4 \tan (k \pi / n)^{2}\right) \lambda_{1} \lambda_{2}<0$, which is always true if (16) holds since $0<\tan (\pi / n) \leq \tan (k \pi / n)<$ $\tan (\pi / 2)$. Therefore, by contradiction, the condition $\left|\phi_{1}-\phi_{2}\right| \neq k \pi, k \in[0,1,2, \ldots\lfloor n / 2\rfloor), \forall \omega^{*} \in$ $\mathbb{R}_{>0}$ can be ensured by proper selection of $\lambda_{1}, \lambda_{2}$. Finally, due to the phase separation property, there always exist a positive constant $\varepsilon$, such that excitation condition (15) is verified for all $t>0$.

Theorem 3.1 (ISS of the adaptive frequency identifier)

Under Assumption 1, given the sinusoidal signal $s(t)$ and the perturbed measurement model (1), the frequency estimation system made up of the two filters (3) of order $n(n \geq 3)$ and by the adaptation law (9) and (10) is ISS with respect to any additive disturbance signal $d(t) \in \mathscr{L}_{\infty}^{1}$ such that

$$
\|d\|_{\infty}<\bar{d}<\gamma_{z}^{\prime-1}\left(\sigma_{1}^{\prime-1}(\varepsilon)\right)
$$

where $\gamma_{z}^{\prime}$ and $\sigma_{1}^{\prime}$ are respectively given by (13) and (14). 
Proof

According to (13), for any positive $\delta^{\prime} \in \mathbb{R}_{>0}$ there exists a finite time-instant $T_{\delta}^{\prime}$ such that $\left|\tilde{\mathbf{z}}^{\prime}(t)\right| \leq$ $\gamma_{z}^{\prime}(\bar{d})+\delta^{\prime}, \forall t \geq T_{\delta}^{\prime}$, which implies

$$
\sigma_{1}^{\prime}\left(\left|\tilde{\mathbf{z}}^{\prime}(t)\right|\right) \leq \sigma_{1}^{\prime}\left(\gamma_{z}^{\prime}(\bar{d})+\delta^{\prime}\right), \quad \forall t \geq T_{\delta}^{\prime}
$$

If the bound (18) on the disturbances holds true, we have that for some $\delta^{\prime} \in \mathbb{R}_{>0}$ the following inequality is satisfied

$$
\varepsilon-\sigma_{1}^{\prime}\left(\gamma_{z}^{\prime}(\bar{d})+\delta^{\prime}\right)>0
$$

Then, for any $t>T_{\delta}^{\prime}$, the following bound on the derivative of candidate Lyapunov function $V=\frac{1}{2} \tilde{\Omega}^{2}$ can be established

$$
\begin{aligned}
\frac{\partial V}{\partial \tilde{\Omega}} \dot{\tilde{\Omega}}(t) & \leq-\mu_{2}\left(\varepsilon-\sigma_{1}^{\prime}\left(\gamma_{z}^{\prime}(\bar{d})+\delta^{\prime}\right)\right)|\tilde{\Omega}(t)|^{2}+\mu_{2} \sigma_{2}^{\prime}\left(\left|\tilde{\mathbf{z}}^{\prime}(t)\right|\right)|\tilde{\Omega}(t)| \\
& \leq-c|\tilde{\Omega}(t)|^{2}+\mu_{2} \sigma_{2}^{\prime}\left(\left|\tilde{\mathbf{z}}^{\prime}(t)\right|\right)|\tilde{\Omega}(t)|, t \geq T_{\delta}
\end{aligned}
$$

where $c \triangleq \mu_{2}\left[\varepsilon-\sigma_{1}^{\prime}\left(\gamma_{z}^{\prime}(\bar{d})+\delta^{\prime}\right)\right]$ is a positive constant. Thus, $\tilde{\Omega}(t)$ is ISS with respect to $\tilde{\mathbf{z}}^{\prime}(t)$ and in turn, ISS with respect to $\bar{d}$.

\subsection{Pre-filter of order $3+3$}

Although the order $n$ of the pre-filter can be set arbitrarily, the use of higher-order filters is not advisable unless needed ${ }^{\dagger}$ due to the decrease in convergence speed and to the excessive attenuation that may lead to numerical issues. To this end, a minimum order scheme (i.e. $3+3$ ) of the kind (9), (10) is preferable on account of such a problem formulation. In this paragraph we briefly describe how a $3+3$ pre-filter-based estimators can be constructed by using the same approach adopted in the generalized $n+n$ case.

The following auxiliary vectors are introduced: $\hat{\mathbf{z}}_{1}^{\prime \prime}(t)=\left[\dot{\hat{x}}_{1,3}(t) \dot{\hat{x}}_{2,3}(t)\right]^{\top}$ and $\hat{\mathbf{z}}_{2}^{\prime \prime}(t)=$ $\left[\dddot{\hat{x}}_{1,3}(t) \dddot{\hat{x}}_{2,3}(t)\right]^{\top}$, formed by the derivatives of the internal pre-filter's states. For simplicity, let us create the combined vector of auxiliary signals $\hat{\mathbf{z}}^{\prime \prime}(t)=\left[\hat{\mathbf{z}}_{1}^{\prime \prime}(t) \hat{\mathbf{z}}_{2}^{\prime \prime}(t)\right]^{\top}$ which can be expressed directly in terms of the available measurement $y(t)$ and the pre-filter's states $\hat{\mathbf{x}}_{k}(t) \in \mathbb{R}^{3}, k=1,2$ :

$$
\hat{\mathbf{z}}^{\prime \prime}(t)=\Lambda_{3}\left[\begin{array}{lll}
y(t) & \hat{\mathbf{x}}_{1}(t)^{\top} & \hat{\mathbf{x}}_{2}(t)^{\top}
\end{array}\right]^{\top},
$$

with

$$
\Lambda_{3}=\left[\begin{array}{ccc}
0 & \mathbf{c}^{\top} \mathbf{F}_{1} & 0 \\
0 & 0 & \mathbf{c}^{\top} \mathbf{F}_{2} \\
\mathbf{c}^{\top} \mathbf{F}_{1}^{2} \mathbf{g}_{1} & \mathbf{c}^{\top} \mathbf{F}_{1}^{3} & 0 \\
\mathbf{c}^{\top} \mathbf{F}_{2}^{2} \mathbf{g}_{2} & 0 & \mathbf{c}^{\top} \mathbf{F}_{2}^{3}
\end{array}\right] .
$$

Finally, the following frequency adaptation law based on the auxiliary signals is proposed:

$$
\dot{\hat{\Omega}}(t)=-\mu_{2} \hat{\mathbf{z}}_{1}^{\prime \prime}(t)^{\top}\left(\hat{\Omega}(t) \hat{\mathbf{z}}_{1}^{\prime \prime}(t)+\hat{\mathbf{z}}_{2}^{\prime \prime}(t)\right)
$$

The stability analysis of the $3+3$ frequency-estimator can be subsumed in the generic $n$-th order case proved in Sec 3.2 and is therefore omitted.

\footnotetext{
$\dagger$ The higher order pre-filtering scheme may turn out to be very useful in the context of parameter estimation problem in presence of higher order structured disturbances (e.g. drift, see [24] and [28]), which is not addressed in this paper. 


\section{METHOD 2: ORDER $2+2$ PREFILTERING-BASED FREQUENCY ESTIMATOR}

\subsection{Underlying idea}

Now, with the aim of further reducing the dynamic order of the estimator, we decrease the order of the two pre-filters to $n=2$. Let us choose $\beta_{1}=\beta_{2}=\beta$ and then introduce the auxiliary signals $z_{1}(t)=x_{1,2}(t)-x_{2,2}(t)$ and $z_{2}(t)=\ddot{x}_{1,2}(t)-\ddot{x}_{2,2}(t)$, it is easy to show that both $z_{1}$ and $z_{2}$ tend asymptotically to a sinusoidal stationary equilibrium:

$$
\begin{aligned}
& z_{1}(t) \stackrel{t \rightarrow \infty}{\longrightarrow} \bar{z}_{1}(t)=A_{1} \sin \left(\vartheta_{1}(t)\right)-A_{2} \sin \left(\vartheta_{2}(t)\right) \\
& z_{2}(t) \stackrel{t \rightarrow \infty}{\longrightarrow} \bar{z}_{2}(t)=-\Omega^{*} \bar{z}_{1}(t)
\end{aligned}
$$

with $\Omega^{*}=\omega^{* 2}$ the true unknown squared-frequency, and where

$$
\begin{aligned}
& A_{k}=A\left|G_{k, 2}\left(j \omega^{*}\right)\right|, \\
& \vartheta_{k}(t)=\vartheta(t)+\angle G_{k, 2}\left(j \omega^{*}\right), k \in\{1,2\},
\end{aligned}
$$

and

$$
G_{k, 2}(s)=\frac{\beta^{2} \lambda_{k}^{2}}{s^{2}+2 \lambda_{k} s+\lambda_{k}^{2}} .
$$

In view of $(24), \bar{z}_{1}(t)$ turns out to be a pure single sinusoidal signal having frequency $\omega^{*}$ and amplitude

$$
A_{z_{1}}=\sqrt{\zeta_{1}^{2}+\zeta_{2}^{2}}
$$

in which

$$
\begin{aligned}
& \zeta_{1}=A_{1} \cos \angle G_{1,2}\left(j \omega^{*}\right)-A_{2} \cos \angle G_{2,2}\left(j \omega^{*}\right) \\
& \zeta_{2}=A_{1} \sin \angle G_{1,2}\left(j \omega^{*}\right)-A_{2} \sin \angle G_{2,2}\left(j \omega^{*}\right) .
\end{aligned}
$$

Similar to the order $n+n$ method, the following gradient-based adaptation law that exploits the available pre-filtered signals $\hat{z}_{1}(t), \hat{z}_{2}(t)$ in place of the unavailable pure sinusoids $\bar{z}_{1}(t)$ and $\bar{z}_{2}(t)$, is adopted for the frequency estimation:

$$
\dot{\hat{\Omega}}(t)=-\mu_{1} \hat{z}_{1}(t)\left(\hat{\Omega}(t) \hat{z}_{1}(t)+\hat{z}_{2}(t)\right)
$$

where $\mu_{1}>0$ is a user-defined adaptation gain aimed at tuning the convergence speed of the estimator.

\subsection{Stability Analysis of the Frequency-adaptation scheme with $2+2$ pre-filter}

For the sake of improving the readability of the stability proof, let us define the combined vector $\hat{\mathbf{z}}(t)=\left[\begin{array}{ll}\hat{z}_{1}(t) & \hat{z}_{2}(t)\end{array}\right]^{\top}$ that can be expressed as

$$
\hat{\mathbf{z}}(t)=\Lambda_{2}\left[\begin{array}{lll}
y(t) & \hat{\mathbf{x}}_{1}(t)^{\top} \quad \hat{\mathbf{x}}_{2}(t)^{\top}
\end{array}\right]^{\top},
$$

where $\hat{\mathbf{x}}_{1}(t)=\left[\begin{array}{ll}\hat{x}_{1,1}(t) & \hat{x}_{1,2}(t)\end{array}\right]^{\top}, \quad \hat{\mathbf{x}}_{2}(t)=\left[\hat{x}_{2,1}(t) \hat{x}_{2,2}(t)\right]^{\top}$ and

$$
\Lambda_{2}=\left[\begin{array}{ccc}
0 & \mathbf{c}^{\top} & -\mathbf{c}^{\top} \\
\mathbf{c}^{\top}\left(\mathbf{F}_{1} \mathbf{g}_{1}-\mathbf{F}_{2} \mathbf{g}_{2}\right) & \mathbf{c}^{\top} \mathbf{F}_{1}^{2} & -\mathbf{c}^{\top} \mathbf{F}_{2}^{2}
\end{array}\right]
$$

Accordingly, the "virtual" combined auxiliary derivatives $\overline{\mathbf{z}}(t)=\left[\bar{z}_{1}(t) \bar{z}_{2}(t)\right]^{\top}$ can be expressed as as

$$
\overline{\mathbf{z}}(t)=\Lambda_{2}\left[\begin{array}{lll}
v(t) & \overline{\mathbf{x}}_{1}(t)^{\top} & \overline{\mathbf{x}}_{2}(t)^{\top}
\end{array}\right]^{\top} .
$$

Furthermore, let $\hat{\mathbf{z}}(t)=\left[\hat{z}_{1}(t) \hat{z}_{2}(t)\right]^{\top}$ and $\tilde{\mathbf{z}}(t)=\hat{\mathbf{z}}(t)-\overline{\mathbf{z}}(t)$ denote the perturbed auxiliary signals and the corresponding error dynamics. Thanks to the ISS of $\tilde{\mathbf{x}}(t)$, the trajectory of $\tilde{\mathbf{z}}(t)$ 
will enter in a closed ball of radius $\gamma_{z}(\bar{d})+\delta$ centered at the origin in finite-time, where

$$
\gamma_{z}(s)=\bar{\lambda}_{2}\left(\gamma_{x}(s)+s\right), \forall s \in \mathbb{R}_{\geq 0}, \delta=\bar{\lambda}_{2} \nu
$$

being $\bar{\lambda}_{2}=\left\|\Lambda_{2}\right\|$. Thanks to the relation $\bar{z}_{2}(t)=-\Omega^{*} \bar{z}_{1}(t)$, let us rewrite (27) in terms of the errors $\tilde{z}_{1}$ and $\tilde{z}_{2}$ :

$$
\begin{aligned}
\dot{\tilde{\Omega}}(t) & =-\mu_{1}\left(\bar{z}_{1}(t)+\tilde{z}_{1}(t)\right)\left(\hat{\Omega}(t)\left(\bar{z}_{1}(t)+\tilde{z}_{1}(t)\right)-\Omega^{*} \bar{z}_{1}(t)+\tilde{z}_{2}(t)\right) \\
& =-\mu_{1} \tilde{\Omega} \bar{z}_{1}(t)^{2}+\mu_{1} \tilde{\Omega} f_{1}(t, \tilde{\mathbf{z}})+\mu_{1} f_{2}(t, \tilde{\mathbf{z}})
\end{aligned}
$$

where

$$
\begin{aligned}
& f_{1}(t, \tilde{\mathbf{z}})=-2 \bar{z}_{1}(t) \tilde{z}_{1}(t)-\tilde{z}_{1}(t)^{2} \\
& f_{2}(t, \tilde{\mathbf{z}})=\Omega^{*}\left(f_{1}(t, \tilde{\mathbf{z}})+\tilde{z}_{1}(t) \bar{z}_{1}(t)\right)-\left(\bar{z}_{1}(t)+\tilde{z}_{1}(t)\right) \tilde{z}_{2}(t) .
\end{aligned}
$$

There also exist two $\mathcal{K}_{\infty}$-functions $\sigma_{1}(\cdot)$ and $\sigma_{2}(\cdot)$ such that

$$
\left|f_{1}(t, \tilde{\mathbf{z}})\right| \leq \sigma_{1}(|\tilde{\mathbf{z}}|), \quad\left|f_{2}(t, \tilde{\mathbf{z}})\right| \leq \sigma_{2}(|\tilde{\mathbf{z}}|) .
$$

The following result enables us to guarantee the stability of the frequency estimator.

Theorem 4.1 (ISS of the adaptive frequency identifier)

Given the sinusoidal signal $v(t)$ and the perturbed measurement model (1), let the bound on the measurement disturbance $d$ verify the inequality:

$$
\|d\|_{\infty}<\bar{d}<\gamma_{z}^{-1}\left(\sigma_{1}^{-1}\left(\frac{\omega^{*} \kappa A_{z_{1}}^{2} \ln \left(\left(1-\kappa_{\Delta}\right) / \kappa\right)}{\mu_{1} \pi \kappa A_{z_{1}}^{2}-2 \omega^{*} \ln (1 / \kappa)}\right)\right)
$$

where $A_{z_{1}}$ is given by $(26), \kappa: \kappa \in(0,1)$ is chosen arbitrarily, $\kappa_{\Delta} \in \mathbb{R}_{>0}$ such that $1-\kappa_{\Delta}>\kappa$ and $\mu_{1}$ is chosen large enough to verify the following two inequalities

$$
\omega^{*} \ln (1 / \kappa) /\left(\mu_{1} \kappa A_{z_{1}}^{2}\right)<\pi
$$

and

$$
\cos ^{2}\left(\frac{\omega^{*} \ln (1 / \kappa)}{2 \mu_{1} \kappa A_{z_{1}}^{2}}\right)-\kappa \geq \frac{\omega^{*} \kappa \ln \left(\left(1-\kappa_{\Delta}\right) / \kappa\right)}{\mu_{1} \pi \kappa A_{z_{1}}^{2}-2 \omega^{*} \ln (1 / \kappa)}
$$

Then, the frequency estimation system given by the two filters (4) with $n=2$ and by (27) and (28) is ISS with respect to $d(t)$.

Proof

Consider the following candidate Lyapunov function $V(\tilde{\Omega})=\frac{1}{2} \tilde{\Omega}^{2}$. In view of (31) and (32), the time-derivative of $V(t)$ along the system's trajectory subsumes the following inequality:

$$
\begin{aligned}
\frac{\partial V}{\partial \tilde{\Omega}} \dot{\hat{\Omega}}(t) & =\tilde{\Omega}(t) \dot{\tilde{\Omega}}(t) \\
& \leq-\mu_{1}\left(\bar{z}_{1}(t)^{2}-\sigma_{1}(|\tilde{\mathbf{z}}(t)|)\right) \tilde{\Omega}(t)^{2}+\mu_{1} \sigma_{2}(|\tilde{\mathbf{z}}(t)|)|\tilde{\Omega}(t)|
\end{aligned}
$$

in which the stationary sinusoidal signal $\bar{z}_{1}(t)$ appears explicitly. At this point, under the assumption of $\bar{d}$ given by (33) and the inequalities (34) and (35), then the period of the squared sinusoid $\bar{z}_{1}^{2}(t)$ can be partitioned in three intervals: $P_{2}$, in which it holds that $\left(\bar{z}_{1}(t)^{2}-\sigma_{1}\left(\gamma_{z}(\bar{d})+\delta\right)\right) \geq \kappa A_{z_{1}}^{2}$ and $P_{1}, P_{3}$, in which this inequality is not guaranteed. In the following, we will denote by $t_{0}, t_{1}$ and $t_{2}$ the transition instants between the aforementioned modes of behavior, as described in Fig.1. We will prove that if the interval $P_{2}$ lasts for more than a specified $\bar{T}_{\epsilon}$, then the discrete-time Lyapunov function obtained by sampling the continuous-time Lyapunov function at the end of the three phases is a discrete-ISS Lyapunov function. Moreover, we will show that the minimum duration $\bar{T}_{e}$ of $P_{2}$ can be guaranteed if the disturbance verifies inequality (33) reported in the statement of the theorem. 


\section{Time $[\mathrm{s}]$}

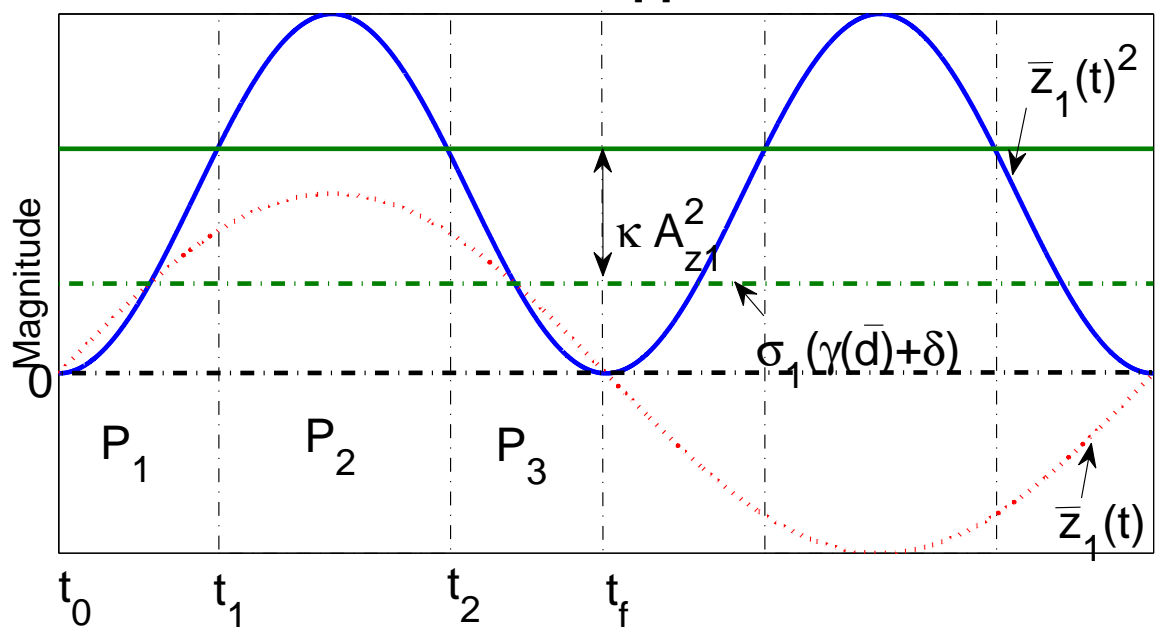

Figure 1. An example plot of the excitation signal $\bar{z}_{1}^{2}(t)$ (blue line) induced by the stationary sinusoidal signal $\bar{z}_{1}(t)$ with amplitude $A_{z_{1}}$ (dotted red line), as well as two horizontal thresholds $\sigma_{1}\left(\gamma_{z}(\bar{d})+\delta\right)$ (dotted green line) and $\sigma_{1}\left(\gamma_{z}(\bar{d})+\delta\right)+\kappa A_{z_{1}}^{2}$ (green line)

During $P_{2}$, we have:

$$
\dot{V}(t) \leq-\mu_{1} \kappa A_{z_{1}}^{2} \tilde{\Omega}(t)^{2}+\mu_{1} \sigma_{2}(|\tilde{\mathbf{z}}(t)|)|\tilde{\Omega}(t)|
$$

By completing squares we get:

$$
\begin{aligned}
\dot{V}(t) & \leq-\mu_{1} \frac{\kappa A_{z_{1}}^{2}}{2} \tilde{\Omega}(t)^{2}+\frac{\mu_{1}}{2 \kappa A_{z_{1}}^{2}} \sigma_{2}(|\tilde{\mathbf{z}}(t)|)^{2} \\
& \leq-\mu_{1} \kappa A_{z_{1}}^{2}\left(V(t)-\sigma_{e}(\bar{d})\right)
\end{aligned}
$$

with $\sigma_{e}(\bar{d})=\left(2 \kappa^{2} A_{z_{1}}^{4}\right)^{-1} \sigma_{2}(|\tilde{\mathbf{z}}(t)|)^{2}$. Analogously, during $P_{1}$ and $P_{3}$, for a time $T_{d}$, with $T_{d}=$ $\frac{\pi}{2 \omega^{*}}-\frac{T_{\epsilon}}{2}$, we obtain the following upper bound for $\dot{V}(t)$

$$
\dot{V}(t) \leq \mu_{1} \sigma_{1}(|\tilde{\mathbf{z}}(t)|)\left(V(t)+\sigma_{d}(\bar{d})\right)
$$

where $\sigma_{d}(s)$ is a $\mathcal{K}$-function such that $\sigma_{d}(s) \sigma_{1}(s)^{2} \geq \frac{\sigma_{2}(s)^{2}}{2}, \forall s \geq 0$.

Applying the Gronwall-Bellman Lemma to (38), the value of the Lyapunov function during $P_{2}\left(t_{1} \leq t<t_{2}\right)$ can be bounded as follows:

$$
\begin{aligned}
V(t) & \leq V\left(t_{1}\right)+\left(1-e^{-\mu_{1} \kappa A_{z_{1}}^{2}\left(t-t_{1}\right)}\right)\left(\sigma_{e}(\bar{d})-V\left(t_{1}\right)\right) \\
& =e^{-\mu_{1} \kappa A_{z_{1}}^{2}\left(t-t_{1}\right)} V\left(t_{1}\right)+\sigma_{e}(\bar{d})\left(1-e^{-\mu_{1} \kappa A_{z_{1}}^{2}\left(t-t_{1}\right)}\right), \forall t \in\left[t_{1}, t_{2}\right)
\end{aligned}
$$

Taking the same steps as above, we obtain a further bound of $V(t)$ during the dis-excited intervals $P_{1}$ and $P_{3}$ :

$$
\begin{aligned}
V(t) \leq V\left(t_{p}\right) e^{\mu_{1} \sigma_{1}(|\tilde{\mathbf{z}}(t)|)\left(t-t_{p}\right)}+\left(e^{\mu_{1} \sigma_{1}(|\tilde{\mathbf{z}}(t)|)\left(t-t_{p}\right)}-1\right) \sigma_{d}(\bar{d}), & \\
& \forall t \in\left[t_{0}, t_{1}\right), \text { if } p=0 \text { or } \forall t \in\left[t_{2}, t_{0}+\frac{\pi}{\omega^{*}}\right), \text { if } p=2
\end{aligned}
$$

Due to the poor excitation during $P_{1}$ and $P_{3}$, at the end of these intervals we can establish a conservative bound

$$
\begin{aligned}
V\left(t_{e}\right) \leq e^{\mu_{1} \sigma_{1}(|\tilde{\mathbf{z}}(t)|) T_{d}}\left(V\left(t_{p}\right)+\sigma_{d}(\bar{d})\right), t_{e}=t_{1}, & \text { if } p=0\left(\text { i.e., in } P_{1}\right) \\
& \text { or } t_{e}=t_{0}+\frac{\pi}{\omega^{*}}, \text { if } p=2\left(\text { i.e., in } P_{3}\right)
\end{aligned}
$$


Then, in view of (40), assuming that the mode $P_{2}$ occurs for a time $T_{e}$ (we do not pose limits on $T_{e}$ at this point, eventually $T_{e}=0$ ), we get the inequality:

$$
V\left(t_{2}\right) \leq e^{-\mu_{1} \kappa A_{z_{1}}^{2} T_{\epsilon}}\left[e^{\mu_{1} \sigma_{1}(|\tilde{\mathbf{z}}(t)|) T_{d}}\left(V\left(t_{0}\right)+\sigma_{d}(\bar{d})\right)\right]+\sigma_{e}(\bar{d})\left(1-e^{-\mu_{1} \kappa A_{z_{1}}^{2} T_{\epsilon}}\right)
$$

Finally, denoting by $V_{k} \triangleq V\left(t_{0}+k \frac{\pi}{\omega^{*}}\right)$ the value of the Lyapunov function at the end of each $k$-th $P_{3}$ interval, and considering that $t_{0}$ is arbitrary within the set $t_{0} \in\left\{t: \bar{z}_{1}^{2}(t)=0\right\}$, from inequalities (42) and (43), we can readily derive the following indexed expression

$$
\begin{aligned}
V_{k+1} \leq e^{2 \mu_{1} \sigma_{1}(|\tilde{\mathbf{z}}(t)|) T_{d}-\mu_{1} \kappa A_{z_{1}}^{2} T_{\epsilon}} V_{k}+ & \sigma_{e}(\bar{d})\left(1-e^{-\mu_{1} \kappa A_{z_{1}}^{2} T_{\epsilon}}\right) e^{\mu_{1} \sigma_{1}(|\tilde{\mathbf{z}}(t)|) T_{d}} \\
& +\left(e^{2 \mu_{1} \sigma_{1}(|\tilde{\mathbf{z}}(t)|) T_{d}-\mu_{1} \kappa A_{z_{1}}^{2} T_{\epsilon}}+e^{\mu_{1} \sigma_{1}(|\tilde{\mathbf{z}}(t)|) T_{d}}\right) \sigma_{d}(\bar{d})
\end{aligned}
$$

In the following lines, we will show that a minimum time-duration of phase $P_{2}$, denoted by $\bar{T}_{\epsilon}$, is ensured as long as the bound (33) is verified. Indeed (33) implies that

$$
\begin{aligned}
\sigma_{1}\left(\gamma_{z}(\bar{d})+\delta\right) & <\frac{\omega^{*} \kappa A_{z_{1}}^{2} \ln \left(\left(1-\kappa_{\Delta}\right) / \kappa\right)}{\mu_{1} \pi \kappa A_{z_{1}}^{2}-2 \omega^{*} \ln (1 / \kappa)} \\
& =\frac{\ln \left(\left(1-\kappa_{\Delta}\right) / \kappa\right)}{2 \mu_{1}\left(\pi / 2 \omega^{*}-\ln (1 / \kappa) / \mu_{1} \kappa A_{z_{1}}^{2}\right)}
\end{aligned}
$$

Then, thanks to (35), there exists a positive constant $\bar{T}_{d}$ that bounds the length of the dis-excitation interval $\left(T_{d} \leq \bar{T}_{d}\right)$ :

$$
\bar{T}_{d} \triangleq \min \left\{t \leq \frac{\pi}{\omega^{*}}: \bar{z}_{1}\left(t_{0}+t\right)^{2} \geq A_{z_{1}}^{2} \cos ^{2}\left(\frac{\omega^{*} \ln (1 / \kappa)}{2 \mu_{1} \kappa A_{z_{1}}^{2}}\right)\right\} .
$$

Owing to the fact that $\bar{z}_{1}\left(t_{0}+\bar{T}_{d}\right)^{2}=A_{z_{1}}^{2} \sin ^{2}\left(\omega^{*}\left(t_{0}+\bar{T}_{d}\right)\right)$, then $\bar{T}_{d}$ can be computed as the minimum positive solution of the equation:

$$
A_{z_{1}}^{2} \sin ^{2}\left(\omega^{*} \bar{T}_{d}\right)=A_{z_{1}}^{2} \cos ^{2}\left(\frac{\omega^{*} \ln (1 / \kappa)}{2 \mu_{1} \kappa A_{z_{1}}^{2}}\right)
$$

From (34), it holds that

$$
\bar{T}_{d}=\frac{\pi}{2 \omega^{*}}-\frac{\ln (1 / \kappa)}{2 \mu_{1} \kappa A_{z_{1}}^{2}}
$$

Hence,

$$
\begin{aligned}
T_{\epsilon} & \geq \frac{\pi}{\omega^{*}}-2 \bar{T}_{d} \\
& \geq \frac{\ln (1 / \kappa)}{\mu_{1} \kappa A_{z_{1}}^{2}}=\bar{T}_{\epsilon} .
\end{aligned}
$$

Combining $\bar{T}_{d}$ and (45), we also have that

$$
e^{2 \mu_{1} \sigma_{1}(|\tilde{\mathbf{z}}(t)|) T_{d}}<e^{2 \mu_{1} \sigma_{1}(|\tilde{\mathbf{z}}(t)|) \bar{T}_{d}}<\frac{\left(1-\kappa_{\Delta}\right)}{\kappa} .
$$

Next, we will prove that the discrete-time system emerging from sampling the Lyapunov function at the transition time instants is ISS with respect to the measurement disturbance. Since $t_{2}-t_{1}=$ $T_{\epsilon} \geq \frac{\ln (1 / \kappa)}{\mu_{1} \kappa A_{z_{1}}^{2}}$, picking an $\epsilon \in \mathbb{R}_{>0}$ such that $1-\kappa_{\Delta}<\epsilon<1$, then we can guarantee the following difference bound on the discrete (sampled) Lyapunov function sequence:

$$
V_{k+1} \leq \epsilon V_{k}+\sigma_{e}(\bar{d})(1-\kappa) \frac{1}{\sqrt{\kappa}}+\left(1+\frac{1}{\sqrt{\kappa}}\right) \sigma_{d}(\bar{d})
$$


which leads to the following compact form:

$$
V_{k+1}-V_{k} \leq-(1-\epsilon) V_{k}+\sigma(\bar{d})
$$

where the function $\sigma(\cdot)$ is $\mathcal{K}$-function defined as

$$
\sigma(s)=\sigma_{e}(s)(1-\kappa) \frac{1}{\sqrt{\kappa}}+\left(1+\frac{1}{\sqrt{\kappa}}\right) \sigma_{d}(s), \forall s \in \mathbb{R}_{\geq 0}
$$

Now, we can conclude that the discrete dynamics induced by sampling the frequency estimator in correspondence of the transitions is Input-to-State stable (ISS).

Finally, we will recover the ISS for the continuous-time system by using the continuity of $V(t)$ and the boundedness of its time-derivative in the inter-sampling. Thanks to the periodicity of the excitation signal $\bar{z}_{1}(t)^{2}$, let us denote by $t_{0}(k), t_{1}(k), t_{2}(k)$ the transition time-instants of the $k$-th period of $\bar{z}_{1}(t)^{2}$, and $k(t)$ the index of the current period: $k(t)=k: t \in\left[t_{0}(k), t_{0}(k+1)\right)$. Between two samples, the Lyapunov function can be bounded conservatively by,

$$
\begin{aligned}
V(t) \leq \frac{1}{\sqrt{\kappa}}\left[V\left(t_{0}\right)+\sigma_{d}(\bar{d})\right]+ & {\left[\sqrt{\kappa}\left(V\left(t_{0}\right)+\sigma_{d}(\bar{d})\right)+\sigma_{e}(\bar{d})\right] } \\
+ & {\left[V\left(t_{0}\right)+\frac{1}{\sqrt{\kappa}} \sigma_{e}(\bar{d})+\left(1+\frac{1}{\sqrt{\kappa}}\right) \sigma_{d}(\bar{d})\right] } \\
= & \frac{1+\sqrt{\kappa}+\kappa}{\sqrt{\kappa}} V_{k(t)}+\left(1+\frac{1}{\sqrt{\kappa}}\right) \sigma_{e}(\bar{d})+\left(2+\frac{2}{\sqrt{\kappa}}\right) \sigma_{d}(\bar{d})
\end{aligned}
$$

Since $k(t) \stackrel{t \rightarrow \infty}{\longrightarrow} \infty$, an infinite number of excited phases with length $T_{\epsilon}$ occurs asymptotically, and then the estimation error in the inter-sampling times converges to a compact region whose radius depends on the assumed disturbance bound.

\section{AMPLITUDE AND PHASE ESTIMATION}

Now, we propose a unified algorithm for the estimation of the amplitude and the phase that applies to all the $n+n(n \geq 2)$ methods presented in this paper. In view of (7), by exploiting the estimated frequency $\hat{\omega}(t)$ obtained by one of the methods described in this paper, the amplitude and phase $\left(A_{k, q}, \vartheta_{k, q}\right)$ of the $q$-th derivative of the $n$-th state variable of the $k$-th pre-filter (see (7) and (8)) can be recovered by

$$
\begin{array}{r}
\hat{A}_{k, q}(t)=\sqrt{\left(\hat{\Omega}(t)\left(\frac{d^{q}}{d t^{q}} x_{k, n}(t)\right)^{2}+\left(\frac{d^{q+1}}{d t^{q+1}} x_{k, n}(t)\right)^{2}\right) / \hat{\Omega}(t)} \\
\hat{\vartheta}_{k, q}(t)=\angle \frac{d^{q+1}}{d t^{q+1}} x_{k, n}(t)+j \hat{\omega}(t) \frac{d^{q}}{d t^{q}} x_{k, n}(t), \quad \forall q \leq n-1
\end{array}
$$

Owing to the asymptotic sinusoidal behavior emerged in (7) and (8), for $t>>0$ we get:

$$
\begin{gathered}
\hat{A}(t)=\frac{\hat{A}_{k, q}(t)}{\hat{\omega}(t)^{q}}\left[\sqrt{\lambda_{k}^{2}+\hat{\omega}(t)^{2}} /\left(\beta_{k} \lambda_{k}\right)\right]^{n} \\
\hat{\vartheta}(t)=\hat{\vartheta}_{k, q}(t)+n \arctan \left(\hat{\omega}(t) / \lambda_{k}\right)-\frac{\pi}{2} q .
\end{gathered}
$$

Note that (47a) and (48a) are "ill" defined at the time instants such that $\hat{\omega}(t)=0$. In order to remove this limitation, the non-adaptive formula for the amplitude described in (47a) is amended by an 
adaptive mechanism:

$$
\dot{\hat{A}}_{k, q}(t)=-\rho \hat{\omega}(t)\left[\hat{\omega}(t) \hat{A}_{k, q}(t)-\sqrt{\left(\hat{\Omega}(t)\left(\frac{d^{q}}{d t^{q}} x_{k, n}(t)\right)^{2}+\left(\frac{d^{q+1}}{d t^{q+1}} x_{k, n}(t)\right)^{2}\right)}\right]
$$

where $\hat{A}_{k, q}(0)=0$ and $\rho \in \mathbb{R}_{>0}$ is the tuning gain fixed by the designers. From (48a), the original amplitude of the sinusoid is adaptively estimated by

$$
\dot{\hat{A}}(t)=-\rho \hat{\omega}(t)^{q}\left[\hat{\omega}(t)^{q} \hat{A}(t)-\hat{A}_{k, q}(t)\left[\sqrt{\lambda_{k}^{2}+\hat{\omega}(t)^{2}} /\left(\beta_{k} \lambda_{k}\right)\right]^{n}\right]
$$

with initial condition $\hat{A}(0)=A_{0}>0$. The above adaption laws makes it easy to enforce, simply by clipping, the positivity constraints $\hat{A}_{k, q}(t)>0$ and $\hat{A}>0$.

\section{SIMULATION AND EXPERIMENTAL RESULTS}

\subsection{Simulation Results}

In this subsection, the behaviour of the proposed methods will be examined and compared with three recent AFP techniques proposed in [11], [13] and [18] respectively. All the algorithms are discretized by Forward-Euler discretization method with fixed sampling period $1 \times 10^{-3} \mathrm{~s}$. The algorithms considered in the comparative analysis have been tuned to have the same response time when fed by a unitary-amplitude sinusoid of frequency $1 /(2 \pi)$, when initialized with zero initial conditions (indeed, the initial transient of the frequency-estimates shown in Fig. 2 put in evidence that the considered methods approximately share the same rise-time). The evaluations are conducted in the case of a biased sinusoidal signal, undergoing both frequency and offset steps-wise variations.

Let us consider a sinusoidal measurement corrupted by a bounded disturbance:

$$
y(t)=\sigma(t)+3 \sin (\omega(t) t+\pi / 4)+d(t),
$$

with

$$
\omega(t)=\left\{\begin{array}{l}
4,0 \leq t<10 \\
8,10 \leq t<35 \\
235 \leq t<50
\end{array} \quad \sigma(t)=\left\{\begin{array}{l}
1,0 \leq t<20 \\
320 \leq t<50
\end{array}\right.\right.
$$

$d(t)$ is a random noise with uniform distribution in the interval [-0.5, 0.5]. All the methods are initialized with the same initial condition $\hat{\omega}(0)=1$. Method [11] is tuned with: $\mu_{0}=1.5, \mu_{1}=$ $3, \mu_{2}=3, \mu_{3}=0.8$, while method [13] is tuned with: $K_{s}=1, \lambda=1, \omega_{s}=4, Q_{0}=(1 / \lambda) \mathbf{I}$. For method [18], we set $\gamma_{0}=\lambda_{0}^{2}=8, \gamma_{1}=6, k=0.18$. Finally, the tuning parameters of the proposed methods are selected by: $\lambda_{1}=6, \lambda_{2}=3, \beta_{1}=0.6, \beta_{2}=0.8, \mu_{2}=1$ and $\lambda_{1}=9, \lambda_{2}=$ $2, \beta_{1}=\beta_{2}=0.6, \mu_{1}=4$ respectively. The real-time frequency-estimation trends obtained by the simulations are depicted in Fig.2.

All the methods are capable to track the initial frequency satisfactorily, with similar response time. The PLL method [11] exhibits a good noise immunity in stationary conditions, but is quite slow to track frequency variations, specially in the low-frequency range. The AFP method [13] is the more sensitive to the bias change, however, it performs the best noise attenuation at the cost of slow reaction to a significant frequency change. The proposed methods can handle considerable frequency variations with relatively faster response time and acceptable robustness against noise. Compared to the single pre-filter-based method [18] (note that the switching dynamic consists in this algorithm is switched off in order to consider the time-varying frequency), in this example the presented methods equipped with parallel pre-filters show certain improvements in terms of noise immunity without losing any transient performance.

The amplitude estimates of each method are reported in Fig.3 ([18] is excluded, since it only deals with frequency estimation). Among the three recursive algorithms, the PLL method [11] is the 


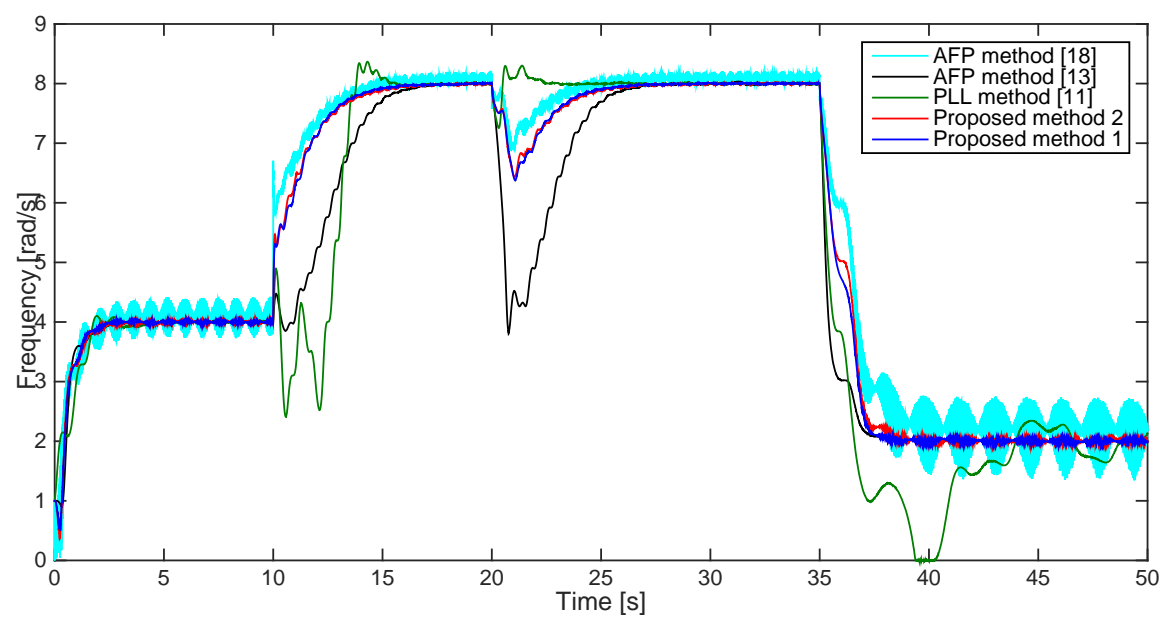

Figure 2. Time-behavior of the estimated frequency by using the proposed AFP methods (blue and red line respectively) compared with the time behaviors of the estimated frequency by the AFP methods [13] (black line), [11] (green line) and [18] (cyan line).

simplest in terms of dynamic order, but it is prone to be disturbed by the parametric perturbations. On the other hand, the proposed methods estimate the amplitude in a relatively smoother manner, in particular, method 1 (order $3+3$ ) provides the best noise immunity in the stationary phases.

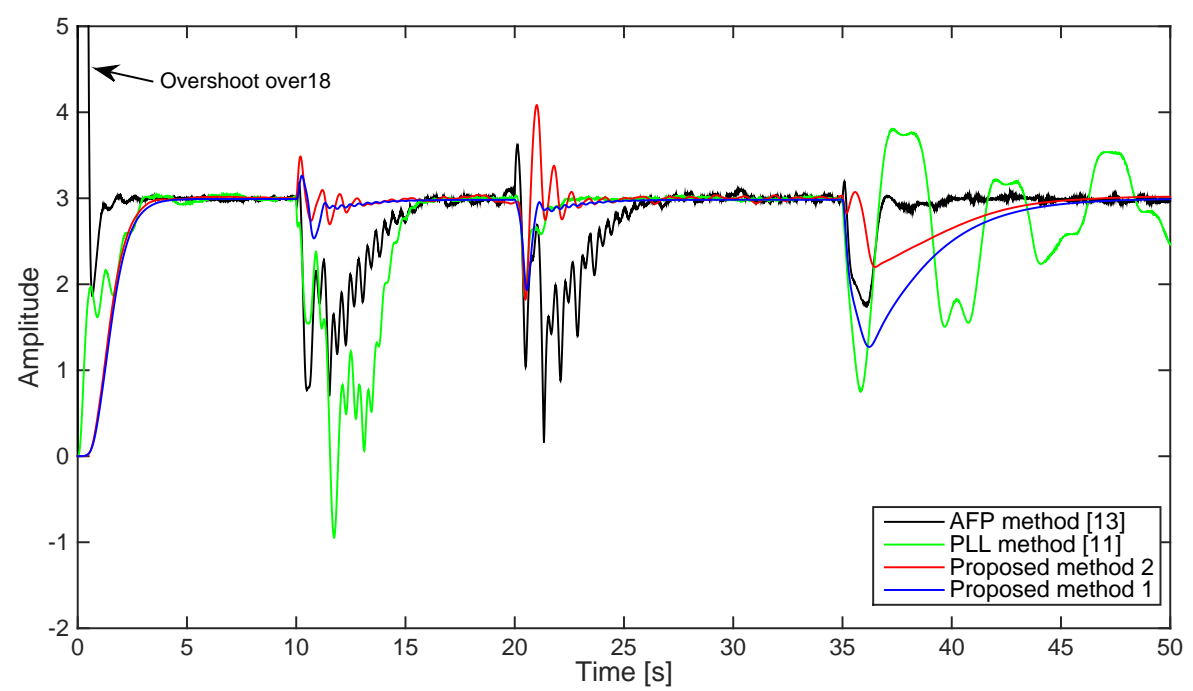

Figure 3. Time-behavior of the estimated amplitude by using the proposed AFP methods (blue and red line respectively) compared with the time behaviors of the estimated frequency by the AFP methods [13] (black line) and [11] (green line).

In order to complete the comparison of the proposed methods, in Fig.4, the time-behaviors of the estimated frequency are shown in another case in which higher level of noise affects the input. It can be observed that method 1 offers slightly better steady state performance at the expense of increased complexity. 


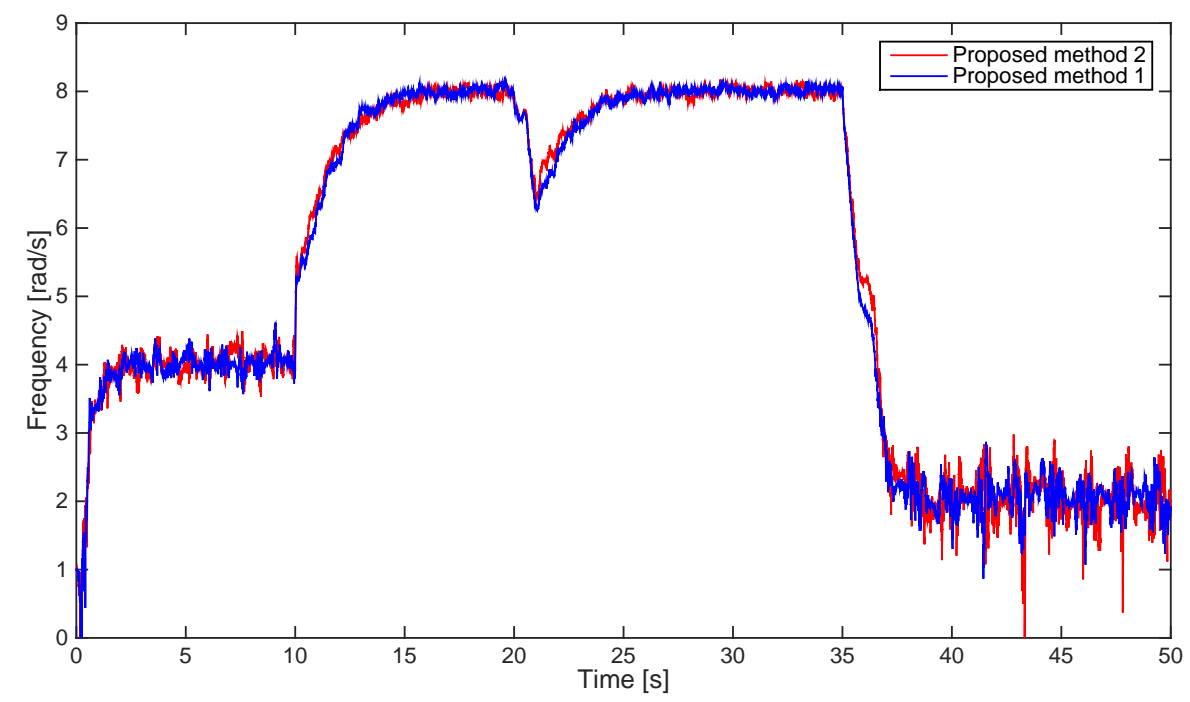

Figure 4. Comparison of the behaviors of the proposed AFP method 1 (blue line) and 2 (red line) in presence of random noise with uniform distribution in the interval $[-5,5]$.

\subsection{Experimental Results}

In order to evaluate the robustness of the proposed algorithm in practice, we have deployed the Method 2 (prefilter order 2+2) on a dSpace system. In order to confirm the simulation results on an experimental test-rig, the two prior methods [11] and [13] have been implemented in the same system, with the task of estimating the frequency, the phase and amplitude of an electrical voltage signal generated by a programmable power supply able to reproduce the perturbations typically experienced in micro-grids. Note that all the methodologies are discretized by Euler method with sampling rate set to $60 \mathrm{kHz}$. Fig. 5 show some periods of the noisy sinusoidal signal generated by the programmable power supply. The large voltage ripples superimposed on the measured sinusoid correspond to the perturbations that may arise from the switching actions of grid-connected power electronic devices. On the other side, voltage-injected spikes are used to reproduce the large voltage transients due to the sudden change of electric loads or to severe RF interference such as those caused by lightning.

In the first test, the frequency of the supply (see Fig. 5) is varied according to the following pattern: $50-48-50-52-48 \mathrm{~Hz}$ (which is likely to occur on a small-scale power system such as a micro-grid). In particular, the frequency changes are composed of multiple steps in order to imitate the inertia of electro-mechanical generators. The tuning gains of the methods [11] and [13] are set to the following values: $\mu_{0}=15, \mu_{1}=\mu_{2}=300, \mu_{3}=0.07$ and $K_{s}=1, \lambda=10, \omega_{s}=2 \pi 50, Q_{0}=(1 / \lambda) \mathbf{I}$, while the parameters of the proposed estimator are: $\lambda_{1}=320, \lambda_{2}=250, \beta_{1}=\beta_{2}=0.6, \mu_{1}=50$. The $\mathrm{dSpace}$ board computes the frequency estimates in real-time and converts them to analog signals that are collected by an oscilloscope whose plot is shown in Fig. 6. The estimates show that all the methods can be adjusted to achieve a similar response time to the given frequency step-wise variations.

For the sake of completeness, with the fixed parameter setting the robustness against bias variations is tested by introducing a measurement offset with step-wise changes $(0-0.5-0)$ over the ac voltage signal given in Fig. 5 . According to the results shown in Fig. 7, the proposed method shows an enhanced transient behavior with only minor loss on the stationary accuracy compared with the other two approaches. 


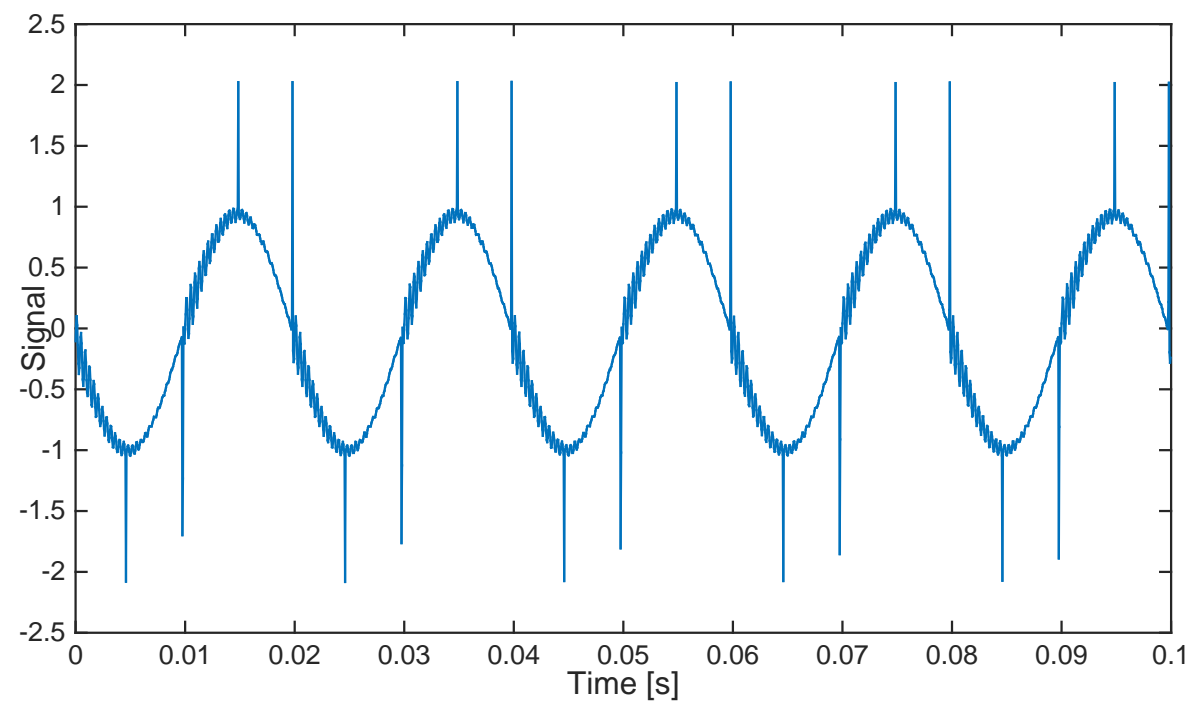

Figure 5. A real-time $50 \mathrm{~Hz}$ sinusoidal voltage signal corrupted by ripples reproducing the perturbation due to a typical switching device and 4 large spikes per cycle to reproduce RF interference.

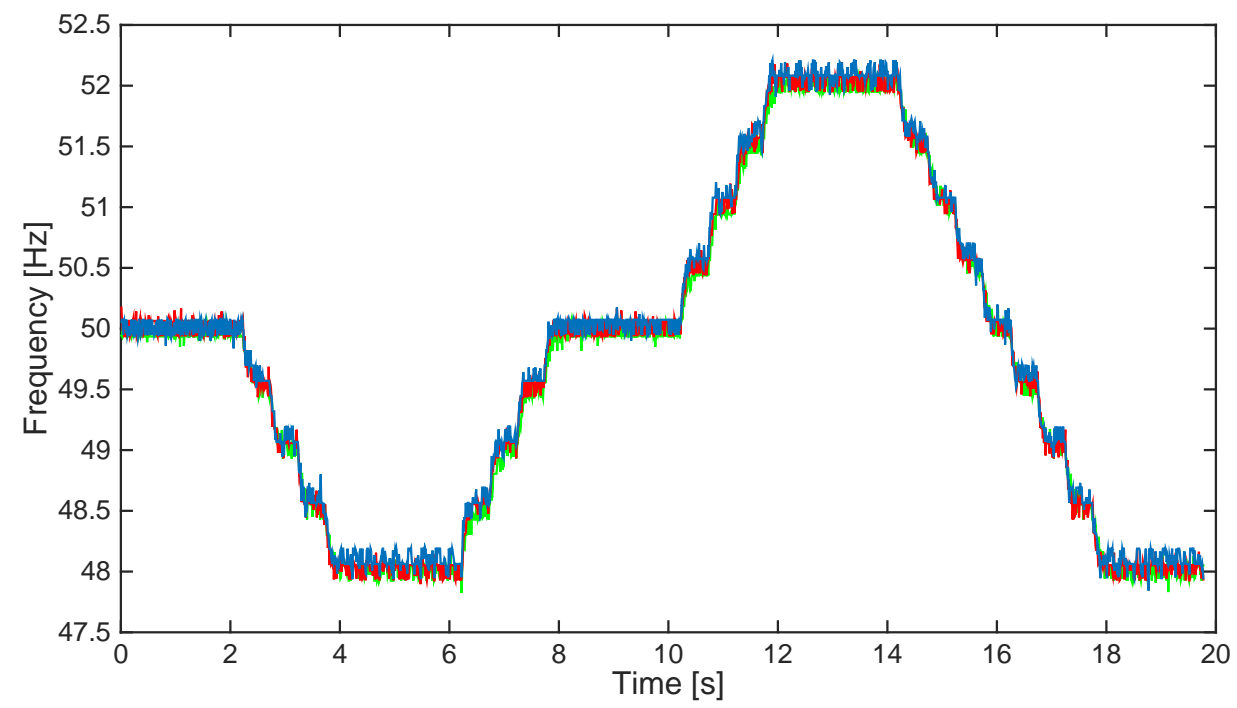

Figure 6. Real-time frequency tracking of a sinusoidal single with a step-wise changing frequency (50-4850-52-48 Hz): the time behaviors of the estimated frequency by the AFP methods [13] (green line), [11] (red line) and the proposed method 2 (blue lines).

\section{CONCLUDING REMARKS}

In this paper, a family of AFP schemes based on the parallel pre-filtering paradigm is proposed. The parallel pre-filtering methodology consists in filtering a noisy-biased sinusoid with a pair of two low-pass filters of a given order, whose inner states are used to estimate the frequency, the phase and the amplitude of the original sinusoid. The robust stability properties of the presented methods with respect to additive measurement perturbations are characterized by an Input-to-State stability analysis, which also provides basic tuning rules for the parameters of the adaptation laws. 


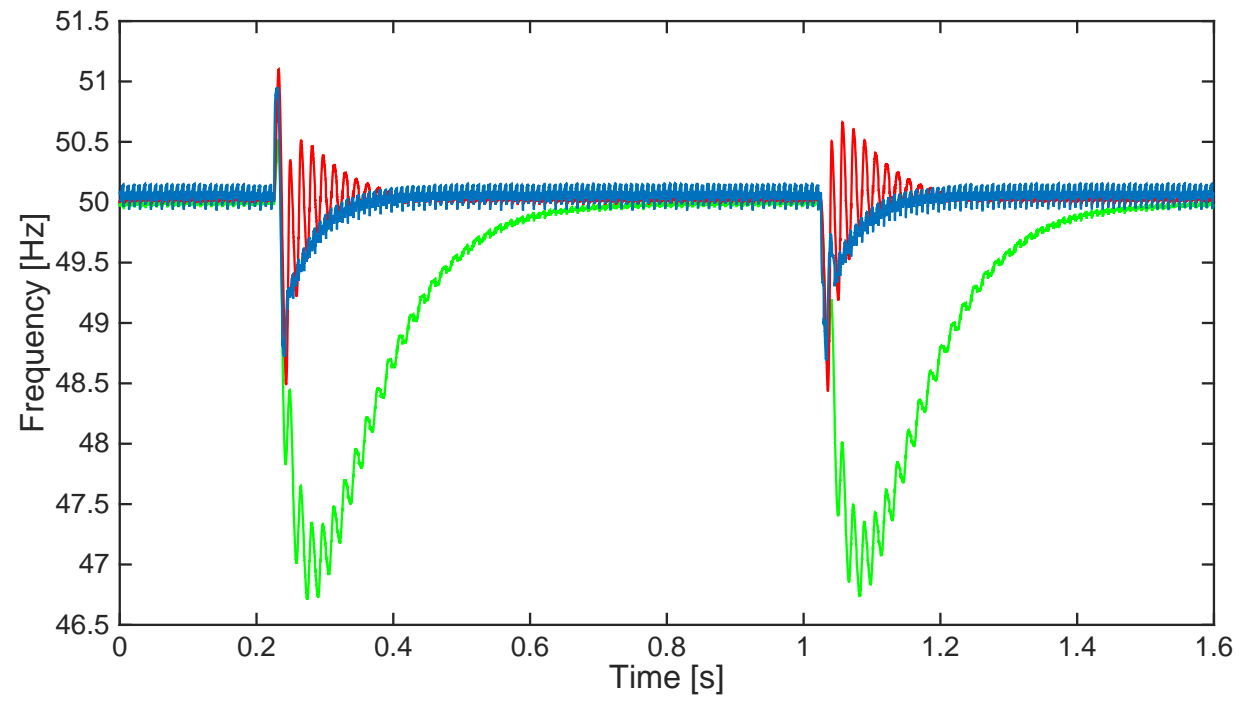

Figure 7. Real-time frequency tracking of a sinusoidal single with a step-wise changing offset: the time behaviors of the estimated frequency by the AFP methods [13] (green line), [11] (red line) and the proposed method 2 (blue lines).

Numerical simulations and practical experiments on a laboratory test-rig confirm that the proposed method is robust with respect to additive measurement noise and is able to track large and sudden frequency variations. Finally, the ease of implementation of the pre-filter-based method makes it a valid alternative to existing AFP methodologies for the task of electrical network monitoring and synchronization with mains.

\section{REFERENCES}

1. M. Bodson and S. Douglas, "Adaptive algorithms for the rejection of sinusoidal disturbances with unknown frequency," Automatica, vol. 33, no. 12, pp. 2213-2221, 1997.

2. R. Marino, G. L. Santosuosso, and P. Tomei, "Robust adaptive compensation of biased sinusoidal disturbances with unknown frequency," Automatica, vol. 39, pp. 1755-1761, 2003.

3. L. J. Brown and Q. Zhang, "Periodic disturbance cancellation with uncertain frequency," Automatica, vol. 40, no. 4, pp. 631-637, 2004.

4. G. Pin, "A direct approach for the frequency-adaptive feedforward cancellation of harmonic disturbances," IEEE Trans. on Signal Processing, vol. 58, no. 7, pp. 3513-3530, 2010.

5. G. Fedele and A. Ferrise, "Biased sinusoidal disturbance compensation with unknown frequency," IEEE Trans. on Automatic Control, vol. 58, no. 12, pp. 3207-3212, 2013.

6. S. Bittanti, M. Campi, and S. Savaresi, "Unbiased estimation of a sinusoid in colored noise via adapted notch filters," Automatica, vol. 33, no. 2, pp. 209-215, 1997.

7. A. K. Ziarani and A. Konrad, "A method of extraction of nonstationary sinusoids," Signal Processing, vol. 84, no. 8, pp. 1323-1346, 2004.

8. M. Karimi-Ghartemani and A. K. Ziarani, "A nonlinear time-frequency analysis method," IEEE Trans. on Signal Processing, vol. 52, no. 6, pp. 1585-1595, 2004.

9. B. Wu and M. Bodson, "A magnitude/phase-locked loop approach to parameter estimation of periodic signals," IEEE Trans. on Automatic Control, vol. 48, no. 4, pp. 612-618, 2003.

10. M. Mojiri and A. R. Bakhshai, "An adaptive notch filter for frequency estimation of a periodic signal," IEEE Trans. on Automatic Control, vol. 49, no. 2, pp. 314-318, 2004.

11. M. Karimi-Ghartemani, S. A. Khajehoddin, P. K. Jain, A. Bakhshai, and M. Mojiri, "Addressing dc component in pll and notch filter algorithms," IEEE Trans. on Power Electronics, vol. 27, no. 1, pp. 78-86, 2012.

12. G. Pin, M. Karimi-Ghartemani, B. Chen, and T. Parisini, "Sinusoidal signal estimation from a noisy-biased measurement by an enhanced pll with generalized error filtering," in Proc. of the IEEE Conf. on Decision and Control, Los Angeles, 2014.

13. G. Fedele and A. Ferrise, "Non adaptive second order generalized integrator for identification of a biased sinusoidal signal," IEEE Trans. on Automatic Control, vol. 57, no. 7, pp. 1838-1842, 2012.

14. _ _ "A frequency-locked-loop filter for biased multi-sinusoidal estimation," IEEE Trans. on Signal Processing, vol. 62, no. 5, pp. 1125-1134, 2014. 
15. G. Fedele, A. Ferrise, and P. Muraca, "An adaptive quasi-notch filter for a biased sinusoidal signal estimation," in IEEE International Conference on Control and Automation (ICCA), Santiago, 2011, pp. 1060-1065.

16. G. Fedele and L.Coluccio, "A recursive scheme for frequency estimation using the modulating function method," Applied Mathematics and Computation, vol. 216, no. 5, pp. 1393-1400, 2010.

17. A. A. Bobtsov, D. Efimov, A. A. Pyrkin, and A. Zolghadri, "Switched algorithm for frequency estimation with noise rejection," IEEE Trans. on Automatic Control, vol. 57, no. 9, pp. 2400-2404, 2012.

18. A. A. Pyrkin, A. A. Bobtsov, D. Efimov, and A. Zolghadri, "Frequency estimation for periodical signal with noise in finite time," in Proc. of the IEEE Conf. on Decision and Control, Orlando, FL, 2011, pp. 3646-3651.

19. R. Marino and P.Tomei, "Global estimation of n unknown frequencies," IEEE Trans. on Automatic Control, vol. 47, no. 8 , pp. $1324-1328,2002$

20. X. Xia, "Global frequency estimation using adaptive identifiers," IEEE Trans. on Automatic Control, vol. 47, no. 7, pp. 1188-1193, 2002.

21. M. Hou, "Parameter identification of sinusoids," IEEE Trans. on Automatic Control, vol. 57, no. 2, pp. 467-472, 2012.

22. A. A. Bobtsov, "New approach to the problem of globally convergent frequency estimator," Int. J. of Adaptive Control and Signal Processing, vol. 22, no. 3, pp. 306-317, 2008.

23. B. Chen, G. Pin, and T. Parisini, "Adaptive observer-based sinusoid identification: structured and bounded unstructured measurement disturbances," in Proc. of the IEEE European Control Conference, Zurich, Switzerland, 2013, pp. 2645-2650.

24. B. Chen, G. Pin, W. M. Ng, C. K. Lee, S. Y. R. Hui, and T. Parisini, "An adaptive observer-based switched methodology for the identification of a perturbed sinusoidal signal: Theory and experiments," IEEE Trans. on Signal Processing, vol. 62, no. 24, pp. 6355-6365, 2015.

25. B. Chen, G. Pin, and T. Parisini, "An adaptive observer-based estimator for multi-sinusoidal signals," in Proc. IEEE American Control Conference, Portland, OR, USA, 2014.

26. D. Carnevale and A. Astolfi, "A hybrid observer for frequency estimation of saturated multi-frequency signals," in Proc. of the IEEE Conf. on Decision and Control and European Control Conference, Orlando, FL, USA, 2011, pp. 2577-2582.

27. G. Pin, T. Parisini, and M. Bodson, "Robust parametric identification of sinusoisal signals: an input-to-state stability approach," in Proc. of the IEEE Conf. on Decision and Control, Orlando, FL, 2011, pp. 6104-6109.

28. G. Pin, B. Chen, T. Parisini, and M. Bodson, "Robust sinusoid identification with structured and unstructured measurement uncertainties," IEEE Trans. on Automatic Control, vol. 59, no. 6, pp. 1588-1593, 2014.

29. S. Aranovskiy, A. Bobtsov, A. Kremlev, N. Nikolaev, and O.Slita, "Identification of frequency of biased harmonic signal," European J. Control, vol. 2, pp. 129-139, 2010. 